\title{
EXPERIMENTAL ANALYSIS ON SPATIAL AND CARTESIAN IMPEDANCE CONTROL FOR THE DEXTEROUS DLR/HIT II HAND
}

\author{
Zhaopeng Chen, ${ }^{*}$ Neal Y. Lii, ${ }^{*}$ Thomas Wimböck, ${ }^{*}$ Shaowei Fan, ${ }^{* *}$ Hong Liu, ${ }^{* *}$ and Alin Albu-Schäffer*
}

\begin{abstract}
This paper presents an experimental study on impedance control in both Cartesian and object level with adaptive friction compensation for dexterous robot hand based on joint torque feedback. To adaptively decrease the effects of high friction caused by complex transmission systems and joint coupling, a friction observer is proposed based on the extended Kalman filter (EKF) in this paper. A Cartesian impedance controller is implemented on a multi-fingered dexterous robot hand with identical fingers, based on the modelling of each modular finger. In addition, a flexible $n$-fingered object frame is proposed in this paper, applicable to any finger configuration with three or more fingers $(n \geq 3)$. This enables the design of a 6-DoF spatial impedance controller. Stability of the closedloop system with friction observer is analysed. A position error of less than $0.16^{\circ}$ is achieved using joint impedance control with adaptive friction compensation, which shows significant improvement in performance, as compared to $1.5^{\circ}$ without compensation, and $0.5^{\circ}$ with fixed-parameters friction compensation. Experimental results confirm the improvement in performance for the robot hand with Cartesian impedance control and adaptive joint friction compensation, demonstrating the effectiveness of spatial impedance controller with the proposed object frame and estimation strategy.
\end{abstract}

\section{Key Words}

Dexterous robot hand, friction observer, impedance control, object frame, joint torque

\section{Introduction}

Dexterous manipulation is one of the most important features for humanoid robots. To achieve this goal,

\footnotetext{
* Institute of Robotics and Mechatronics, German Aerospace Center, DLR, Germany; e-mail: zzhaopeng.chen, neal.lii, thomas.wimboeck, alin.albu-schaeffer $\} @$ dlr.de

** State Key Laboratory of Robotics and System, Harbin Institute of Technology, 150001 Harbin, China; e-mail: fansw@hit.edu.cn, dlrhitlab@yahoo.com.cn

Recommended by Dr. P.X. Liu

(DOI: 10.2316/Journal.206.2014.1.206-3669)
}

several anthropomorphic robot hands have been developed in recent decades. The embedded actuator approach, as taken by the Gifu Hand [1], the DLR Hand II [2] and the DLR-HIT Hand I [3], integrate all the actuators inside the hand, fingers or joints, in order to achieve shorter power transmission distances. With a different approach, the Stanford-JPL Hand [4], the Utah/MIT Hand [5], the NASA Robonaut Hand [6] and the DLR Hand Arm System [7] are designed with all the actuators mounted outside the robot fingers driven through tendon cables. Both approaches introduce complex dynamics including high friction, flexibility and joint couplings, which hinder the control performance of the robot hands. In this paper, we introduce the modelling and control strategies of the dexterous robot hand DLR-HIT II, a multisensory five-finger hand jointly designed by German Aerospace Center (DLR) and Harbin Institute of Technology (HIT), with a total of 15 DOFs and more than 100 sensors [8].

Tasks such as grasping and manipulation are essential features for dexterous robotic hands to fully function as end-effectors for humanoid robots. Impedance control frame was introduced by Hogan [9] as a reliable approach for compliant dexterous manipulation and grasping. Impedance control can be realized in Cartesian space as a desired dynamic behaviour on the end-effector motion with respect to the external forces/torques. The robot manipulator impedance control strategy can be separated into two categories: with end-force/torque sensor feedback [10], [11], and with joint torque sensors feedback [12], [13]. The latter approach is mostly utilized on robot arms. Theoretical analyses have been presented, with numerous experimental realization carried out on the DLR Light-Weight Robot Arm series [12], [13].

The former approach has also been implemented on robot hands to achieve Cartesian compliance, known as admittance control [14], [15]. However, the feedback with high level of signal noise from six-dimensional force/torque sensors can significantly hinder performance of the above control strategies. To address this, sufficient experimental analysis on joint torque sensor-based robotic hand with 
lower signal noise is necessary, which is currently unavailable in literature.

Object level impedance control strategies have been intensively investigated on cooperative robotic manipulators [10], [11], [16]-[18]. A passivity-based approach, namely the intrinsically passive controller, is introduced with differentiable manifolds [19]. This approach is implemented on the four-fingered robotic hand DLR II [20]. This work extends the object-level impedance controller concept to accommodate any $n$-fingered $(n \geq 3)$ robotic hand configuration. Implementation and experimental analysis are made on the five-fingered dexterous robotic hand DLR-HIT II.

Nonlinear factors, such as friction, are addressed by using stiffness parameters design, rather than explicitly analysed for joint torque-based robotic arms [12], [13]. Literature on friction models are available [21]-[24], and numerous model-based friction observers have been derived in previous work [25]-[28]. Due to uncertainties of friction model in robot manipulator, disturbance observers are investigated in [29]-[31]. With the embedded-actuator design of DLR-HIT II, limited construction space, transmission system complexity (including harmonic drivers and timing belts), and joint coupling (e.g., bevel gears), can hinder the performance of robot hands due to friction and gravity, more so than robot arms, which have larger distances between adjacent joints. In addition, the joint friction model is difficult to identify explicitly due to the mechanical couplings in the finger joint, as well as the compact design. The parameters of the joint friction may also vary from joint to joint resulting from component tolerances. To address these concerns, we present an extended Kalman filter (EKF) based friction observer derived from a simple friction model with static and viscous terms, to adaptively estimate the joint friction.

In this paper, we propose an adaptive joint friction observer based on the EKF and establishing an object level frame based on $n$-finger positions, so that the Cartesian and 6-DoFs spatial impedance controllers can be implemented and evaluated on a multi-fingered robot hand. Our work distinguishes itself from previous studies in that we not only present an impedance controller with adaptive friction compensation for joint torque-based robotic hand with compact design, but we also present a multi-fingered spatial impedance controller based on $n$-fingered object frame with nonlinear compensation.

\section{The Robot Hand with Flexible Joints}

The fingers of DLR-HIT II, shown in Fig. 1 are of identical, modular designed. Flexibility of the finger joints is modelled as a linear torsional spring with stiffness $k$, the detailed description could be found in [31]. The dynamics equations of the robot finger with flexible joint can be written as [39]:

$$
\begin{gathered}
M(q) \ddot{q}+C(q, \dot{q}) \dot{q}+g(q)=\tau+\tau_{\text {ext }} \\
I \ddot{\theta}_{m}+\tau+\tau_{f, m}=\tau_{m} \\
\tau=K\left(q-\theta_{m}\right)
\end{gathered}
$$

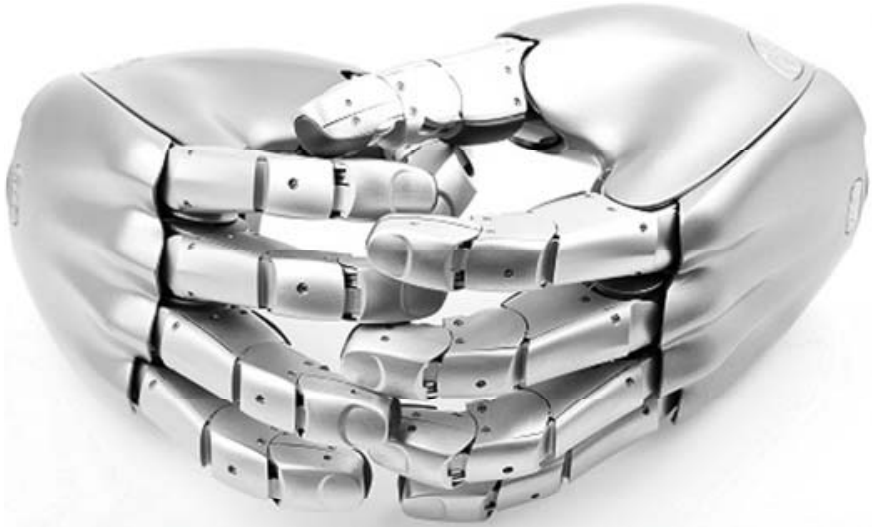

Figure 1. The DLR-HIT II dexterous robot hand.

where $M(q), C(q)$ and $g(q)$ represent the inertia matrices, centrifugal term and gravity term of the entire finger, respectively. The joint torque vector is given by $K\left(q-\theta_{m}\right)$, where $\theta_{m}$ indicates the vector of the motor angle divided by the gear ratio $n$, and $q$ represents the link side joint angle. $K$ and $I$ are diagonal matrices which contain the joint stiffness $k$, and the motor inertia multiplied by the gear ratio squared, respectively. $\tau_{\text {ext }}$ and $\tau_{f, m}$ are external torque vector and friction torque vector in joint space, respectively. The generalized actuator torque vector, $\tau_{m}$, is considered as the control input [33], [34].

\section{Cartesian Impedance Control Design for the Robot Hand}

To obtain the desired impedance relationship, the Cartesian position error $\tilde{x}=x-x_{d}$ is introduced as the difference between the centre point of the fingertip position $x$ and a desired position $x_{d}$. The Cartesian impedance controller is designed to realize a dynamical behaviour between the position error $\tilde{x}$ and the external force $F_{\text {ext }}$ :

$$
F_{e x t}=M_{d} \ddot{\tilde{x}}+D_{d} \dot{\tilde{x}}+K_{d} \tilde{x}
$$

with $\dot{x}=J(q) \dot{q}$, where $J(q)$ represents the Jacobian matrix of the finger. Together with (1) and (4), the Cartesian impedance controller is derived with $F_{\tau}$ considered as the control input:

$$
\begin{aligned}
F_{\tau}= & \Lambda(x) \ddot{x}_{d}+\mu(x, \dot{x}) \dot{x}+\left(\Lambda(x) M_{d}^{-1}-I\right) F_{e x t} \\
& +F_{g}(x)-\Lambda(x) M_{d}^{-1}\left(D_{d} \dot{\tilde{x}}+K_{d} \tilde{x}\right)
\end{aligned}
$$

The matrices $\Lambda(x)$ and $\mu(x, \dot{x})$ here can be expressed as:

$$
\begin{gathered}
\Lambda(x)=J(q)^{-T} M(q) J(q)^{-1} \\
\mu(x, \dot{x})=J(q)^{-T}\left(C(q, \dot{q})-M(q) J(q)^{-1} \dot{J}(q)\right) J(q)^{-1}
\end{gathered}
$$

$F_{\text {ext }}$ and $F_{g}$ are external torques/forces and gravity represented in Cartesian coordinates system. The Coriolis term is considered in the theoretical analysis in this work, in 
order to remain consistent with the dynamics model of the robotic hand. However, since the Coriolis term caused by the limited maximum joint speed of our robot is within a reasonable boundary, the centripetal and Coriolis forces are neglected here in the actual implementation. In addition, the desired inertia $M_{d}$ can be designed to be identical to the finger inertia matrix $\Lambda(x)$, so that $F_{\text {ext }}$ is eliminated in the dynamics equations. As a result, the actual impedance controller in implementation becomes:

$$
F_{\tau}=\Lambda(x) \ddot{x}_{d}+\mu(x, \dot{x}) \dot{x}-D_{d} \dot{\tilde{x}}-K_{d} \tilde{x}+F_{g}(x)
$$

The model-based controller can be represented with $\tau$ as control input in joint space:

$$
u_{c m}=g(q)+J(q)^{T}\left(\Lambda(x) \ddot{x}_{d}+\mu(x, \dot{x}) \dot{x}-D_{d} \dot{\tilde{x}}-K_{d} \tilde{x}\right)
$$

For passivity consideration and simplification of the damping design, impedance controller is chosen as PD control law more explicitly in [12]:

$$
\begin{gathered}
u_{c}=-J(\theta)^{T}\left(K_{x} \tilde{x}(\theta)+D_{x} \dot{x}\right) \\
\tilde{x}(\theta)=f(\theta)-x_{d} \\
\dot{x}=J(\theta) \dot{\theta}
\end{gathered}
$$

Without considering friction term, (2) is rewritten as:

$$
I \ddot{\theta}_{m}+\tau=\tau_{m c}
$$

With the control input $u_{c}$, the joint dynamics is expressed as:

$$
\ddot{\theta}_{m}=-I_{v}^{-1} \tau+I_{v}^{-1} u_{c}
$$

where $I_{v}$ is a diagonal virtual inertia matrix, with all the none zero entries $i_{v}<i$. This means that the robot system with the designed impedance controller appears to react to external forces/torques with a reduced rotor inertia [12]. From (10) and (11), the actual implementation of Cartesian impedance controller can be expressed as:

$$
\tau_{m c}=K_{\tau} u_{c}+\left(I-K_{\tau}\right) \tau
$$

This is a PD-form controller with the torque feedback loop. $K_{\tau}=I \cdot I_{v}^{-1}$ is a diagonal matrix representing the torque feedback gain, which contains $k_{\tau i}>1$. System dynamics terms such as $\Lambda(x), \mu(x, \dot{x})$ and friction $\tau_{f}$ are not compensated for. Dynamics model errors should be considered within the design of the stiffness parameter $K_{d}$ while implementing PD-based impedance control law. Without considering friction, the closed loop system can be rewritten as:

$$
\begin{gathered}
M(q) \ddot{q}+C(q, \dot{q}) \dot{q}+g(q)=\tau+\tau_{\text {ext }} \\
I_{v} \ddot{\theta}_{m}+J(\theta)^{T}\left(K_{x} \tilde{x}(\theta)+D_{x} \dot{x}\right)+\tau=0
\end{gathered}
$$

System dynamics with friction and friction compensation is further investigated in Section 4.

\section{Spatial Impedance Control for the Multi- fingered Dexterous Robot Hand}

Modular and identical design of the DLR-HIT II robot fingers permits constructing $n$-fingered dexterous robot hand depending on tasks. A flexible $n$-fingered object frame is proposed in this section following with spatial impedance controller implementation. The assumption is made in this paper that only the fingertips are in contact with the object while grasping. The point contact with friction (PCWF) contact model without sliding is considered in the remainder of this paper.

The object frame is solely based on the fingertip positions. In contrast to [20], the object frame proposed in this work can be applied to $n$-fingered robot hand configuration with $n \geq 3$. The centre point $x_{o}$ of the object frame is chosen as:

$$
x_{o}=\frac{x_{1}+x_{2}+\cdots+x_{n}}{n}
$$

where $x_{i}(\theta)$ denotes the $i$ th fingertip position represented in the local coordinates. With the assumption presented above, there is no relative velocity between $x_{o}$ and the object centre, although $x_{o}$ may not necessarily coincide with the object centre. As a result, $x_{o}$ can be adopted to represent the object motion in the proposed frame.

The objective of the spatial impedance controller design is to map the object driving force/toque with respect to the object frame into motor control input with respect to the joint frame. Therefore, a connecting vector between the $i$ th fingertip and the frame centre is defined as $\Delta x_{i}=x_{i}-x_{o}$, as shown in Fig. 2. Thus the object frame $\sum o=\left[R_{o}, x_{o}\right] \in S E(3)$ is linear spanned by all of the connecting vectors, in which $R_{o}=\left[r o_{1}, r o_{2}, r o_{3}\right]$ represents the orientation matrix. The first axis $r_{1}(x)$ of $\sum o$ is expressed as:

$$
r o_{1}=\frac{v_{1}+v_{2}}{\left\|v_{1}+v_{2}\right\|}
$$

where:

$$
\begin{gathered}
v_{1}=\sum_{i=0}^{n}(-1)^{i} \frac{x_{2 i+1}-x_{o}}{\left\|x_{2 i+1}-x_{o}\right\|} \\
v_{2}=\sum_{i=1}^{n}(-1)^{(i+1)} \frac{x_{2 i}-x_{o}}{\left\|x_{2 i}-x_{o}\right\|}
\end{gathered}
$$

The third axis $\mathrm{ro}_{3}(z)$ of $\sum o$ is defined perpendicular to the plane spanned by vectors $v_{1}$ and $v_{2}$ :

$$
r o_{3}=\frac{v_{1} \times v_{2}}{\left\|v_{1} \times v_{2}\right\|}
$$

The second axis $\mathrm{ro}_{2}(y)$ of $\sum o$ can therefore be represented as:

$$
r \mathrm{o}_{2}=\mathrm{ro}_{3} \times r \mathrm{O}_{1}
$$




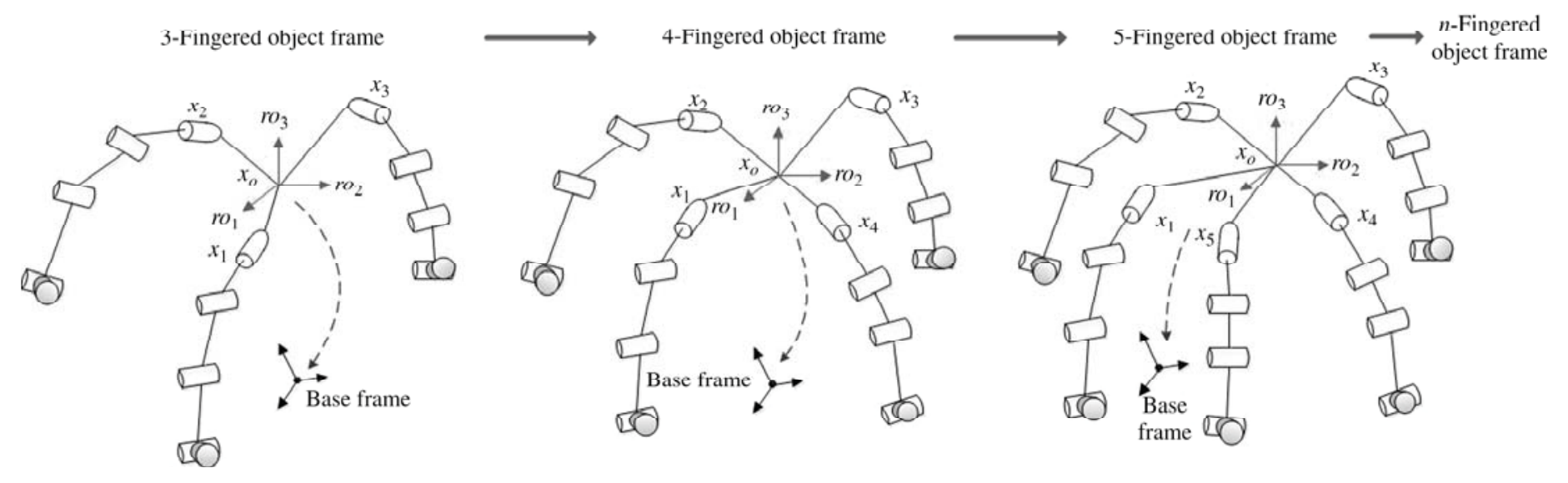

Figure 2. Construction of the object frame based on 3, 4 and 5 fingers configurations with the robot hand DLR-HIT II.

By applying a spatial virtual mechanical spring between the object frame $\sum o$ and the desired virtual equilibrium object frame $\sum d[35]$, the spatial 6-DoFs impedance control can be achieved with $n$ fingers for coordinated control. To maintain stable grasping forces while manipulating the object, an additional virtual connecting spring is implemented as described in [20]. To achieve asymptotic stability, a possible set of energies in the translational, rotational and connecting springs is defined as:

$$
\begin{gathered}
V_{t}=\frac{1}{2} \Delta x_{o d}^{o T} K_{t} \Delta x_{o d}^{o} \\
V_{o}=2 \epsilon_{o d}^{o T} K_{o} \epsilon_{o d}^{o} \\
V_{c}=\frac{1}{2} K_{c} \sum_{i=0}^{n}\left(\left\|\Delta x_{i}\right\|-l_{d i}\right)^{2}
\end{gathered}
$$

with $\Delta x_{o d}^{o}=R_{o}^{T}\left(x_{o}-x_{d}\right)$ denoting object position error represented in object frame. $\epsilon_{o d}^{o}$ is the vector component of the unit quaternion $\left\{\eta_{o d}, \epsilon_{o d}^{b}\right\}$, which can be generated from $R_{d}^{o}=R_{o}^{T} R_{d} . \quad l_{d i}$ is determined depending on the requirements of force closure during grasping. $K_{t}, K_{o}$ and $K_{c}$ are diagonal matrices representing translational, rotational and connecting stiffness parameters, respectively. With the energy derivations from virtual springs, the coordinated spatial impedance control law can be derived:

$$
u_{s}=-\frac{\partial\left(V_{t}+V_{o}+V_{c}\right)}{\partial \theta}-D v_{o}+g(q)
$$

where $D$ is a positive definite damping matrix. $v_{o}$ represents object velocity with respect to $\sum o$. The actual implementation of the spatial impedance controller is achieved with the joint torque feedback:

$$
\tau_{m s}=K_{\tau} u_{s}+\left(I-K_{\tau}\right) \tau
$$

\section{Adaptive Friction Observer}

\subsection{Friction Estimation based on Least Squares}

The performance of the controller is closely tied to the accuracy of the robot dynamic model. Due to the mechanical couplings in the finger joint and the compact design, the joint friction model is difficult to identify explicitly. In addition, the parameters of the joint friction vary from joint to joint as a result of component tolerances. Hence, a conventional friction model is utilized for the joint of robotic hand DLR-HIT II. The friction model is expressed as:

$$
\tau_{f}=b \dot{\theta}+c \cdot \operatorname{sign}(\dot{\theta})
$$

$b$ and $c$ are coefficients of viscous and static friction, respectively, with which the joint dynamics can be described as below:

$$
I \ddot{\theta}=\bar{\tau}-b \dot{\theta}-c \cdot \operatorname{sign}(\theta)
$$

where $\bar{\tau}=\tau_{m}-\tau, I$ represents motor inertia.

The friction compensation is implemented at the joint level in this paper. The friction coefficients in (30) must be identified in order to implement friction compensation. However, the signum function $\operatorname{sign}(\dot{\theta})$ introduces nonlinearity into (30). Linearization of the joint dynamics equation can be achieved by multiplying both sides of (30) with $\dot{\theta}$ :

$$
I_{m} \dot{\theta} \ddot{\theta}+b \dot{\theta}^{2}+c \underbrace{\dot{\theta} \operatorname{sign}(\dot{\theta})}_{|\dot{\theta}|}=\dot{\theta} \tau
$$

The friction coefficient estimations based on Least Squares (LS) technique can be obtained by integrating (31):

$$
\begin{gathered}
\underbrace{\left[\begin{array}{cc}
\frac{1}{2}\left(\dot{\theta}_{k+1}^{2}-\dot{\theta}_{k}^{2}\right) & \frac{t_{s}}{2}\left(\dot{\theta}_{k+1}^{2}+\dot{\theta}_{k}^{2}\right) \\
\downarrow k=0 \ldots N & \frac{t_{s}}{2}\left(\left|\dot{\theta}_{k+1}^{2}\right|+\left|\dot{\theta}_{k}^{2}\right|\right) \\
\vdots
\end{array}\right]}_{L S} \cdot\left[\begin{array}{c}
I_{m} \\
b \\
c
\end{array}\right] \\
=\left[\begin{array}{c}
\frac{t_{s}}{2}\left(\tau_{k+1} \dot{\theta}_{k+1}-\tau_{k} \dot{\theta}_{k}\right) \\
\downarrow k=0 \ldots N \\
\vdots
\end{array}\right]
\end{gathered}
$$


The above integrating is performed over one time step $t_{s}=t_{k+1}-t_{k}$, and trapezoidal approximation is utilized during the integrating step. It should be noted that the velocity $\dot{\theta}$ could not be measured directly. Instead it has to be obtained through the derivation of the position $\theta$, which results in the high frequency noise on the velocity signal. On the other hand, the LS estimation is also influenced by the integral approximation. In order to achieve a more satisfactory estimation result, an adaptive joint friction and velocity observer based on EKF will be derived in the following section.

\subsection{Adaptive Friction Observer based on EKF}

The partially observed nonlinear dynamic system can be expressed as:

$$
\begin{aligned}
& \dot{x}=f(x, u) \\
& y=h(x, u)
\end{aligned}
$$

The EKF for (28) is given by [36]:

$$
\begin{gathered}
\dot{\hat{x}}(t)=f(\hat{x}(t), u(t))+K(t)(y(t)-h(\hat{x}(t), u(t))) \\
\dot{P}(t)= \\
\quad F(t) P(t)+P(t) F^{T}(t)+Q \\
-P(t) H^{T}(t) R^{-1}(t) H(t) P(t)
\end{gathered}
$$

where $F$ and $H$ are:

$$
\begin{aligned}
& F(t)=\frac{\partial f}{\partial x}(\hat{x}(t), u(t)) \\
& H(t)=\frac{\partial h}{\partial x}(\hat{x}(t), u(t))
\end{aligned}
$$

As described in [37] and [38], the viscous and static friction parameters $b$ and $c$ are assumed to be constant system states during the derivation. The control input $\tau_{m}$ is modelled as a state variable, and the external disturbance torque $\tau$ is obtained directly from the torque sensors. With a high sample rate $(1 \mathrm{kHz}), \tau_{m}$ as well as $\tau$ are modelled as constant system variables. As a result, the dynamic model of the robot finger joint can be described as:

$$
\frac{d}{d t} \underbrace{\left(\begin{array}{c}
\theta \\
\dot{\theta} \\
\bar{\tau} \\
b \\
c
\end{array}\right)}_{:=x}=\underbrace{\left(\begin{array}{c}
\dot{\theta} \\
\frac{1}{I}(\bar{\tau}-b \dot{\theta}-c \cdot \operatorname{sign}(\dot{\theta})) \\
0 \\
0 \\
0
\end{array}\right)}_{:=f(x)}
$$

Since the derivation of the sign function in (36) is not defined, the following approximations are used to replace the sign function and its derivation:

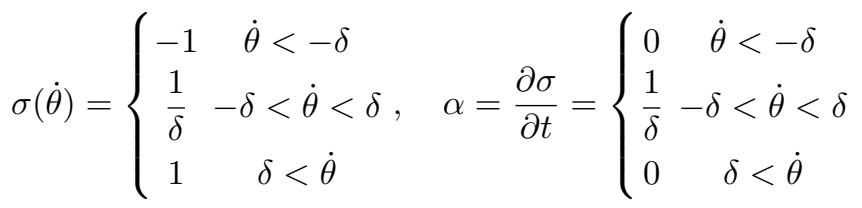

After partially deriving the dynamics (38), the following linearized error state system dynamics can be obtained:

$$
\frac{d}{d t}\left(\begin{array}{c}
\Delta \theta \\
\Delta \dot{\theta} \\
\Delta \bar{\tau} \\
\Delta b \\
\Delta c
\end{array}\right)=\left(\begin{array}{ccccc}
0 & 1 & 0 & 0 & 0 \\
0-\left(\frac{b}{I}+\alpha \frac{c}{I}\right) & \frac{1}{I} & \frac{-\dot{\theta}}{I} & \frac{-f(\dot{\theta})}{I} \\
0 & 0 & 1 & 0 & 0 \\
0 & 0 & 0 & 1 & 0 \\
0 & 0 & 0 & 0 & 1
\end{array}\right) \cdot\left(\begin{array}{c}
\Delta \theta \\
\Delta \dot{\theta} \\
\Delta \tau \\
\Delta b \\
\Delta c
\end{array}\right)
$$

with $\alpha$ represents the derivation of $\sigma(\dot{\theta})$. The partial derivative sign $\partial$ is replaced with the forward difference $\operatorname{sign} \Delta$, and (39) is utilized.

With the adaptive friction observer, the finger joint friction estimation can be expressed as:

$$
\hat{\tau}_{f}=\hat{b} \dot{\theta}+\hat{c} \cdot \operatorname{sign}(\dot{\theta})
$$

The Cartesian and spatial impedance controllers with adaptive friction observer can be written as:

$$
\tau_{m}=K_{\tau} u+\left(I-K_{\tau}\right) \tau+\left(I-K_{\tau}\right) \hat{\tau}_{f}
$$

$u$ represents $u_{c}$ in the Cartesian impedance controller, whereas $u_{s}$ is employed as $u$ in the spatial impedance controller.

\subsection{Gravity Compensation}

By using the iteration method introduced in [12], (10) and (27) with gravity compensation can be rewritten as below:

$$
u_{c}=\hat{g}(\theta)-J(q)^{T}\left(D_{d} \dot{\tilde{x}}+K_{d} \tilde{x}\right)
$$

$$
u_{s}=-\frac{\partial\left(V_{t}+V_{o}+V_{c}\right)}{\partial \theta}-D v_{o}+\hat{g}(\theta)
$$

The link side gravity can be compensated with the motor position [12]. As the gravity force of the joints cannot be explicitly obtained due to the compact design of the finger joints on DLR-HIT II robot hand, the LS technique is utilized during the system identification [39]. 


\section{Stability Discussion with a Passivity-based Controller with Friction Compensation}

The local convergence of the EKF in continuous time is demonstrated in continuous time by Kerner [40], Reif et al. [41], and in discrete time by Song and Grizzle [42], Boutayeb et al. [43]. Following [40], the candidate Lyapunov function including the filter energy is chosen as:

$$
V_{f}=\tilde{x}_{f}(t)^{T} Q(t) \tilde{x}_{f}(t)
$$

where $\tilde{x}_{f}=x-\hat{x}$ refers to the state estimation error. Since $\tilde{x}_{f}(t)^{T} Q(t) \tilde{x}_{f}(t) \rightarrow 0$ exponentially as $t \rightarrow \infty$, as a result:

$$
\dot{V}_{f}=\frac{d}{d t} \tilde{x}_{f}(t)^{T} Q(t) \tilde{x}_{f}(t) \leq 0
$$

Detailed proof of (46) are available in [40] and [41]. The closed-loop system with the passive PD controller (17) is analysed here. Similar stability analysis could be constructed for passivity-based controller (27) and (28). With considering friction and friction compensation, the closedloop system dynamics (17) can be expressed as:

$$
I_{v} \ddot{\theta}+J(\theta)^{T}\left(K_{x} \tilde{x}(\theta)+D_{x} \dot{x}\right)+\tau+\left(\tau_{f}-\hat{\tau}_{f}\right)=0
$$

A candidate Lyapunov function for the closed-loop system is chosen as:

$$
\begin{aligned}
& V=V_{f}+V(q, \dot{q}, \theta, \dot{\theta}) \\
& V(q, \dot{q}, \theta, \dot{\theta})= \frac{1}{2} \dot{q}^{T} M(q) \dot{q}+\frac{1}{2} \dot{\theta}^{T} I_{v} \dot{\theta} \\
&+\frac{1}{2}(\theta-q)^{T} K(\theta-q)+\frac{1}{2} \tilde{x}(\theta)^{T} K_{x} \tilde{x}(\theta) \\
&+V_{g}(q)-V_{\hat{g}}(\theta)
\end{aligned}
$$

where $V(q, \dot{q}, \theta, \dot{\theta})$ represents kinetic and potential energy from the closed-loop system. Following [12], the derivative of $V(q, \dot{q}, \theta, \dot{\theta})$ is expressed as:

$$
\dot{V}(q, \dot{q}, \theta, \dot{\theta})=-\dot{x}^{T} D_{x} \dot{x}+\dot{\theta}\left(\hat{\tau}_{f}-\tau_{f}\right)
$$

The derivative of the Lyapunov function $V$ with the addition of the filter energy, given in (46), becomes:

$$
\dot{V}=-\dot{x}^{T} D_{x} \dot{x}+\dot{\theta}\left(\hat{\tau}_{f}-\tau_{f}\right)+\dot{V}_{f}
$$

Based on the observations of (51), $\dot{V}$ is negative definite with a negative definite $\dot{\theta}\left(\hat{\tau}_{f}-\tau_{f}\right)$ along with an appropriately chosen $D_{x}$. $\dot{\theta}\left(\hat{\tau}_{f}-\tau_{f}\right)$ represents the power introduced by friction compensation. Thus, the condition of negative definite $\dot{\theta}\left(\hat{\tau}_{f}-\tau_{f}\right)$ requires that the power of friction compensation to be dissipative, to reach in the following conclusion:

$$
\dot{V}=-\dot{x}^{T} D_{x} \dot{x}+\dot{\theta}\left(\hat{\tau}_{f}-\tau_{f}\right)+\dot{V}_{f} \leq 0
$$

Due to the local convergence of the observer, the friction observer provides exact friction compensation at steady state, e.g., $\hat{\tau}_{f}-\tau_{f}=0$. Therefore, the system converges to an invariant set contained in subspace, which is given by the following unique point:

$$
P=\left(\theta=\theta_{s}, \dot{\theta}=0, q=q_{s}, \dot{q}=0, v_{m}=0, \hat{\tau}_{f}=\tau_{f}\right)^{T}
$$

where the steady state is derived from (16) and (47):

$$
\begin{gathered}
K\left(\theta_{s}-q_{s}\right)=g\left(q_{s}\right) \\
K\left(\theta_{s}-q_{s}\right)+J\left(\theta_{s}\right)^{T} K_{x} \tilde{x}\left(\theta_{s}\right)+\hat{\tau}_{f}-\tau_{f}=\bar{g}(\theta)
\end{gathered}
$$

As shown by LaSalle Invariance Principle, asymptotic stability is achieved. The analysis is restricted to the condition that the power of friction compensation is dissipative. The above analysis implies that a passivity-based controller for a robot with flexible joints is asymptotically stable with a passive friction observer.

However, the limitation of the EKF-based approach lies in the fact that the power of EKF-based friction compensation is not always dissipative. As a result, (52) does not always hold true. Therefore, a friction compensation gain $K_{f}$ is introduced, and the control law (42) with adjustable friction compensation is given as:

$$
\tau_{m}=K_{\tau} u+\left(I-K_{\tau}\right) \tau+\left(I-K_{\tau}\right) K_{f} \hat{\tau}_{f}
$$

Following [44], a possible energy storage function may be chosen as:

$$
S=\frac{1}{2} \dot{\theta}^{T} I_{v} \dot{\theta}+\frac{1}{2}(\dot{\theta}-\dot{\hat{\theta}})^{T} K_{f}(\dot{\theta}-\dot{\hat{\theta}})
$$

By monitoring the energy introduced by the observer and friction compensation in a period of time $t \rightarrow t_{0}$ :

$$
U_{\text {fric }}=S(t)-S\left(t_{0}\right)-\int_{t_{0}}^{t} \dot{\theta}(u-\tau) \mathrm{d} t
$$

The friction compensation gain $K_{f}$ can be scaled down when $U_{\text {fric }}$ exceeds a certain threshold, in order to eliminate the friction overcompensation.

\section{Experiments}

\subsection{Friction and Velocity Estimation}

Experiments of the friction and velocity estimation with the EKF are carried out with the DLR-HIT II. The finger distal joint of the first robot finger is adopted for experimental investigation. Results are illustrated in Fig. 4, with the finger distal joint moving without external torque $\left(\tau_{\text {ext }}=0\right)$. The estimation of friction coefficients converges to the assumed values $(b=0.3, c=0.2)$ within 80 control cycles, as shown in Fig. 3(a)-(c), which demonstrates the effectiveness of the designed observer based on the EKF. The assumed friction parameters are calculated with the 


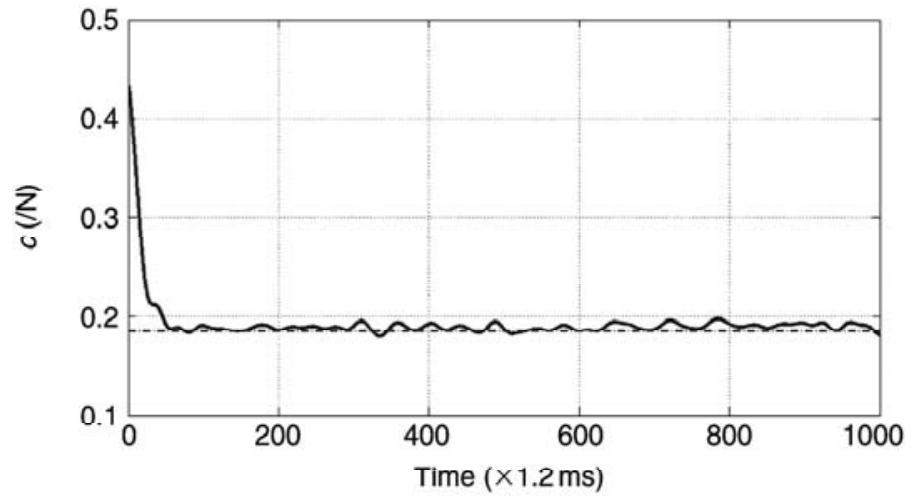

(a)

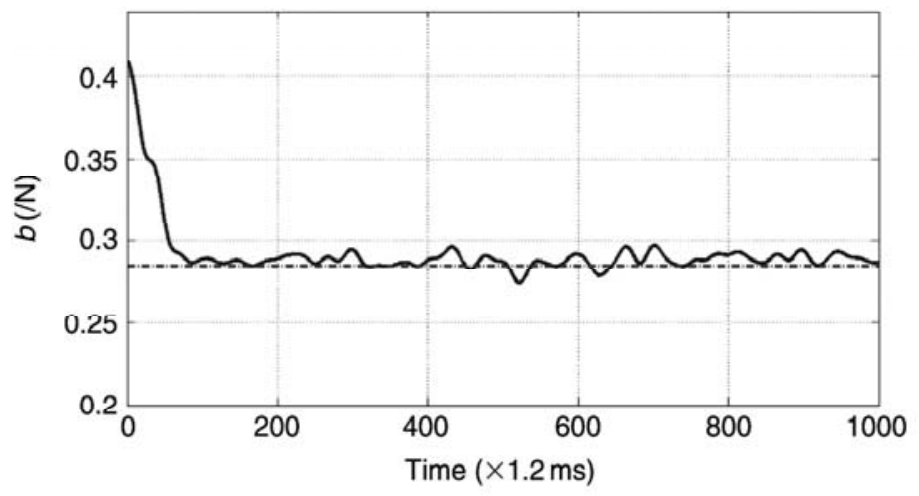

(c)

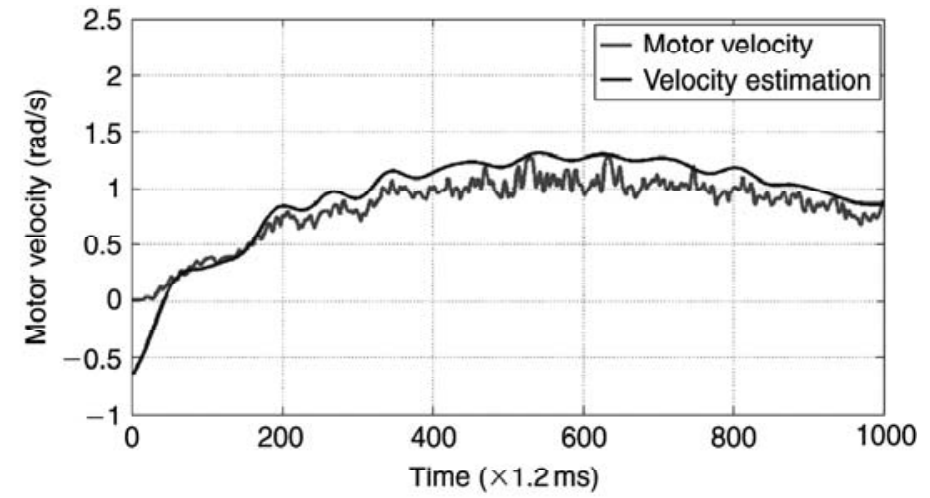

(b)

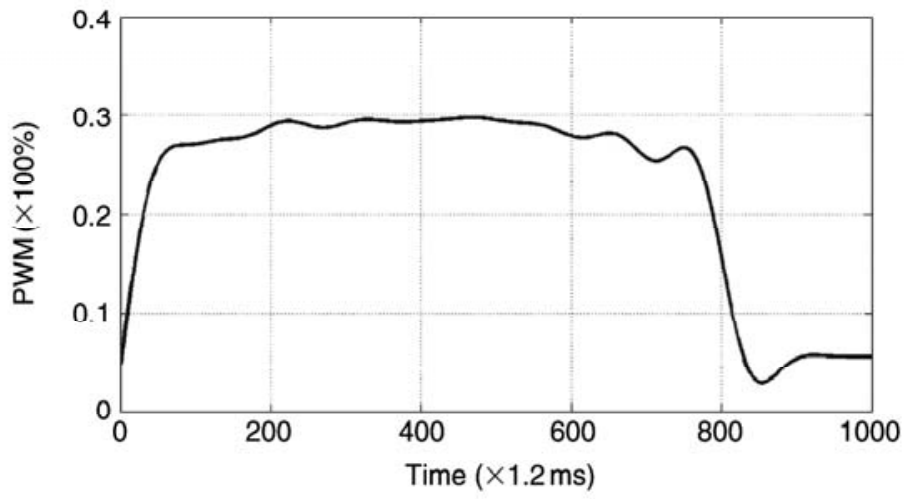

(d)

Figure 3. Friction parameters and velocity estimation with EKF: (a) estimation of static friction coefficient $c$; (b) velocity estimation; (c) estimation of viscous friction coefficient $b$; and (d) control input.

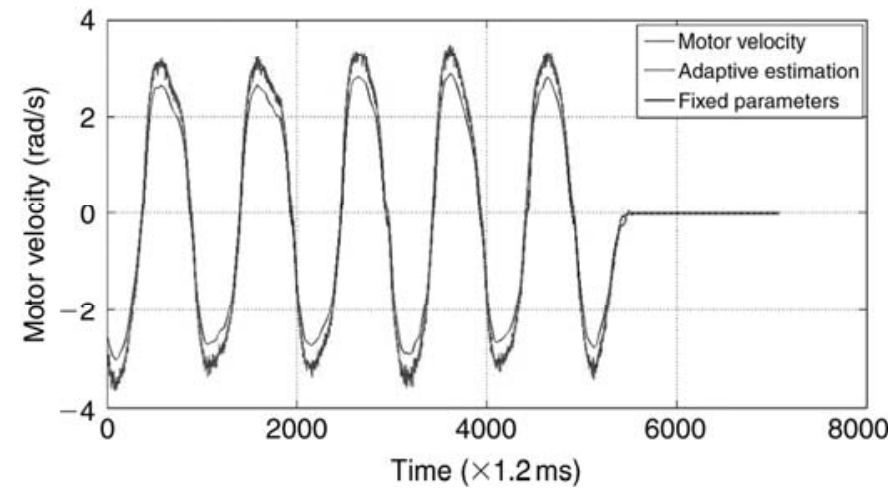

Figure 4. Velocity estimation with EKF and LS method.

LS technique. Control effort is shown in Fig. 3(d). The steady-state errors shown in Fig. 3 may be caused by the approximations during the derivation of the EKF filter. As described in [40], the initial state should be chosen to be sufficiently close to the actual state, such that $|x(t)-\hat{x}(t)| \rightarrow 0$ exponentially as $t \rightarrow 0$. The state variables $\theta, \dot{\theta}$ and $\tau$ are initialized with sensor measured values. The friction parameters are initiated with arbitrary values in an appropriate range such that $b(0), c(0) \in\left[\begin{array}{ll}0.1 & 0.5\end{array}\right]$, in order to show the local convergence property of the EKF.

Higher values of measurement noise covariance $R$ guarantee the local convergence of the EKF, at the expense of slower convergence rate [40]. Therefore, a tradeoff should be made between stability and rate of convergence for the selection of $R$.
During the velocity estimation experiments, the fingertip is pulled and pushed by the operator in order to generate observable velocities. The experimental results on DLR-HIT II robot finger demonstrate robust performance against external disturbance force, as shown in Fig. 4. The velocity estimation with adaptively estimated system parameters shows better results than the velocity estimation with pre-identified system parameters based on LS, which indirectly demonstrate the performance improvement facilitated by the friction observer. The computation time of the EKF-based observer is $160-180 \mu$ s with $1.2 \mathrm{~ms}$ control cycle, in comparison to $1-6 \mu$ s of the LS-based observer, with a QNX real-time OS on Pentium IV PC.

\subsection{Friction Compensation in Joint Space}

Friction compensation experiments are carried out with the impedance controller in joint space, which can be expressed as $u=-K_{d}\left(\theta-\theta_{s}\right)-D_{d} \dot{\theta}$, where $\theta_{s}$ represents the desired configuration. $K_{d}$ and $D_{d}$ denote the desired stiffness and damping parameters, respectively. Table 1 lists the relevant parameters of the robot finger required in the experiment. During experiment, the third joint of the robot finger follows a periodic trajectory generated by a third-order interpolation method. The experimental results are shown in Fig. 5. Together with adaptive friction and optimal gravity compensation, the joint impedance controller shows position tracking error of less than $0.16^{\circ}$. This result compares favourably to position errors of $0.5^{\circ}$ and $1.5^{\circ}$ achieved by joint impedance control with 
Table 1

Estimated and Measured Dynamics Parameters of the DLR-HIT II Modular Robot Finger

\begin{tabular}{|l|c|c|c|c|}
\hline Parameters & $\begin{array}{c}\text { Length } \\
(\mathrm{mm})\end{array}$ & $\begin{array}{c}\text { Estimated Mass } \\
(\mathrm{kg})\end{array}$ & $\begin{array}{c}\text { Mass centre } \\
(\mathrm{mm})\end{array}$ & $\begin{array}{c}\text { Estimated Inertia } \\
\left(\mathrm{kg} \mathrm{mm}^{2}\right)\end{array}$ \\
\hline Joint 1 & 0 & 0.00232 & 27.49 & 17.53 \\
\hline Joint 2 & 55 & 0.0232 & 27.49 & 17.53 \\
\hline Joint 3 & 25 & 0.0158 & 2.5 & 0.098 \\
\hline Joint 4 & 15 & 0.0137 & 3 & 0.1233 \\
\hline
\end{tabular}
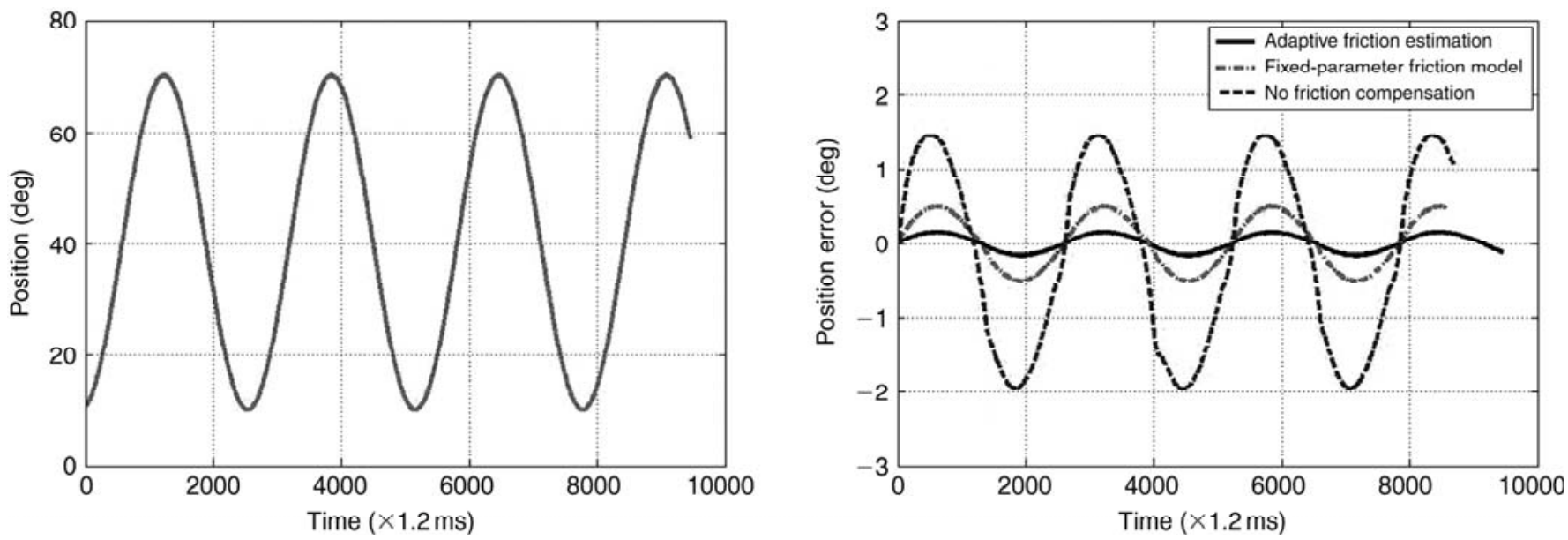

Figure 5. Position tracking experiments with and without friction and gravity compensation.
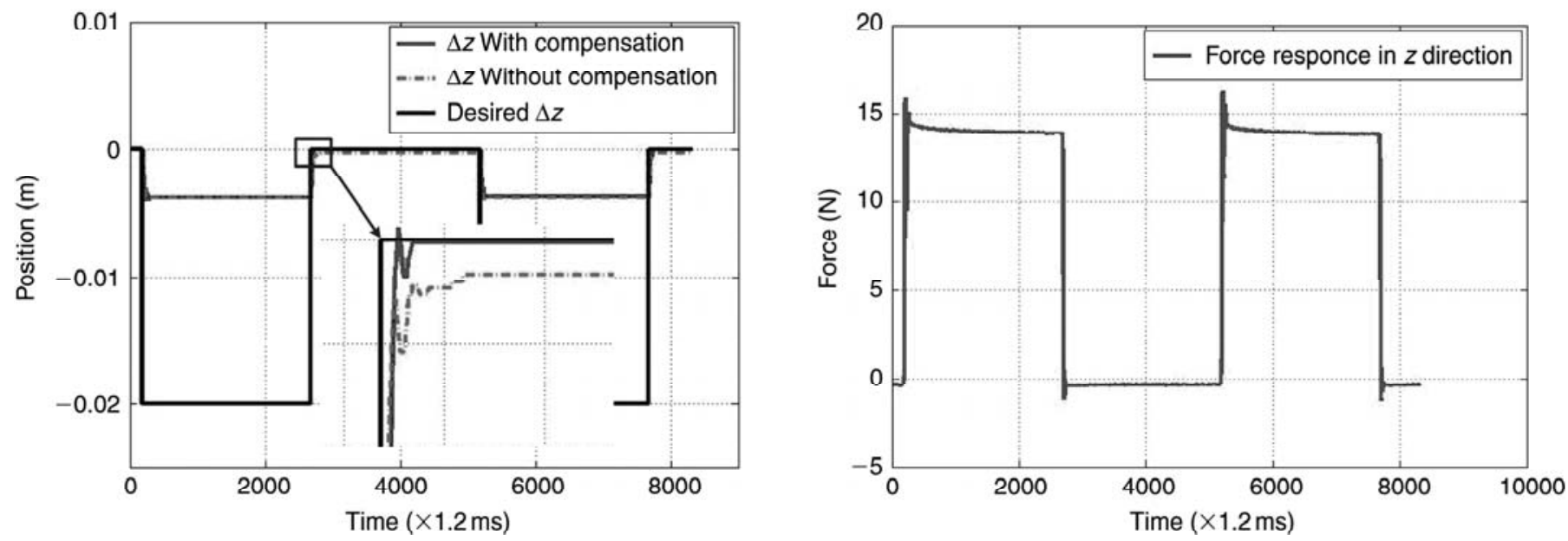

Figure 6. Cartesian position and force responses when making contact with contacting environment.

friction compensation based on pre-identified parameters, and without friction compensation, respectively. We can conclude that improved performance is obtained by the joint impedance controller with the proposed adaptive friction observer.

\subsection{Cartesian Impedance Control on the DLR-HIT II Robot Hand}

The Cartesian impedance control experiments with and without friction compensation have been carried out with the modular robotic finger. $D_{x}=[100,100,100] \mathrm{N} \times \mathrm{s} / \mathrm{m}$ and $K_{k}=[900,900,900] \mathrm{N} / \mathrm{m}$ are calculated through the Double-Diagonalization method [13]. $\Lambda(x)$ and $g(x)$ can be generated by using the estimated inertias and masses in Section 4.1. As shown in Fig. 6, the Cartesian position tracks the desired position trajectory (solid black line), with real tracking curve (solid gray line). Contacts are made with the rigid environment where the position offset is $\Delta z=-0.004 \mathrm{~m}$ in the $z$-direction. The Cartesian force response in $z$-direction can also be found in Fig. 6 . The experimental results show that the Cartesian position can follow the desired trajectory closely in the free space, and the Cartesian force increases stably while staying in contact 

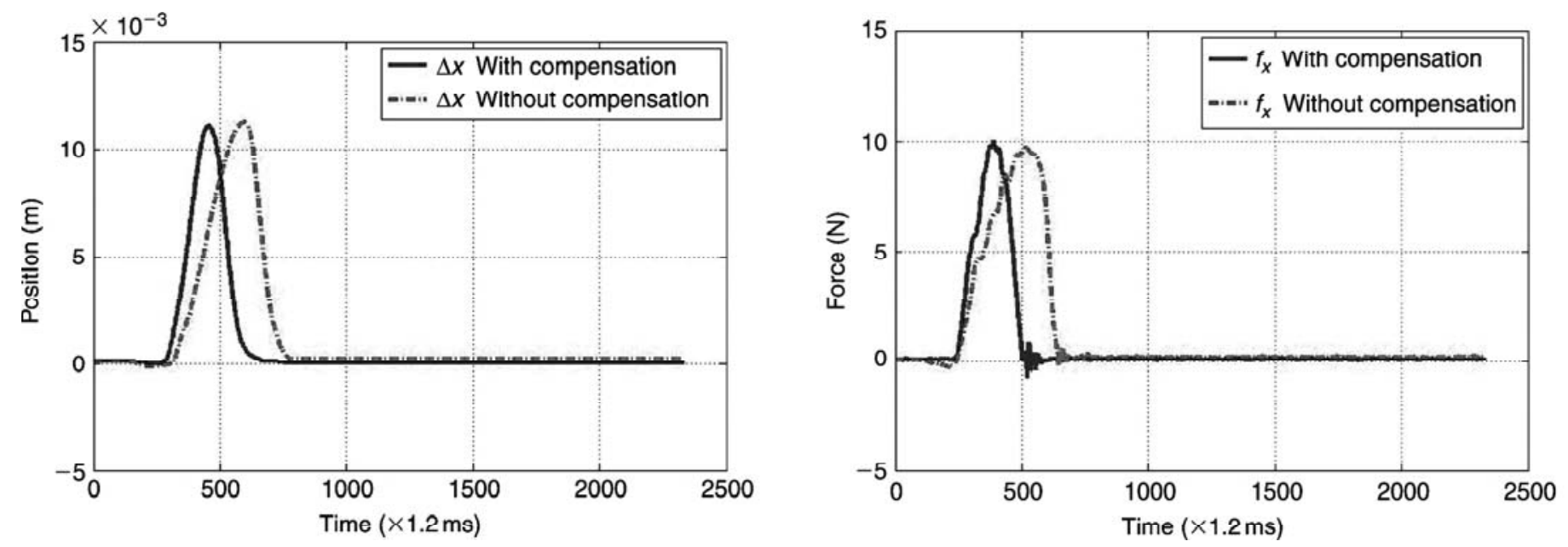

Figure 7. Cartesian position and force responses as an external force is applied.
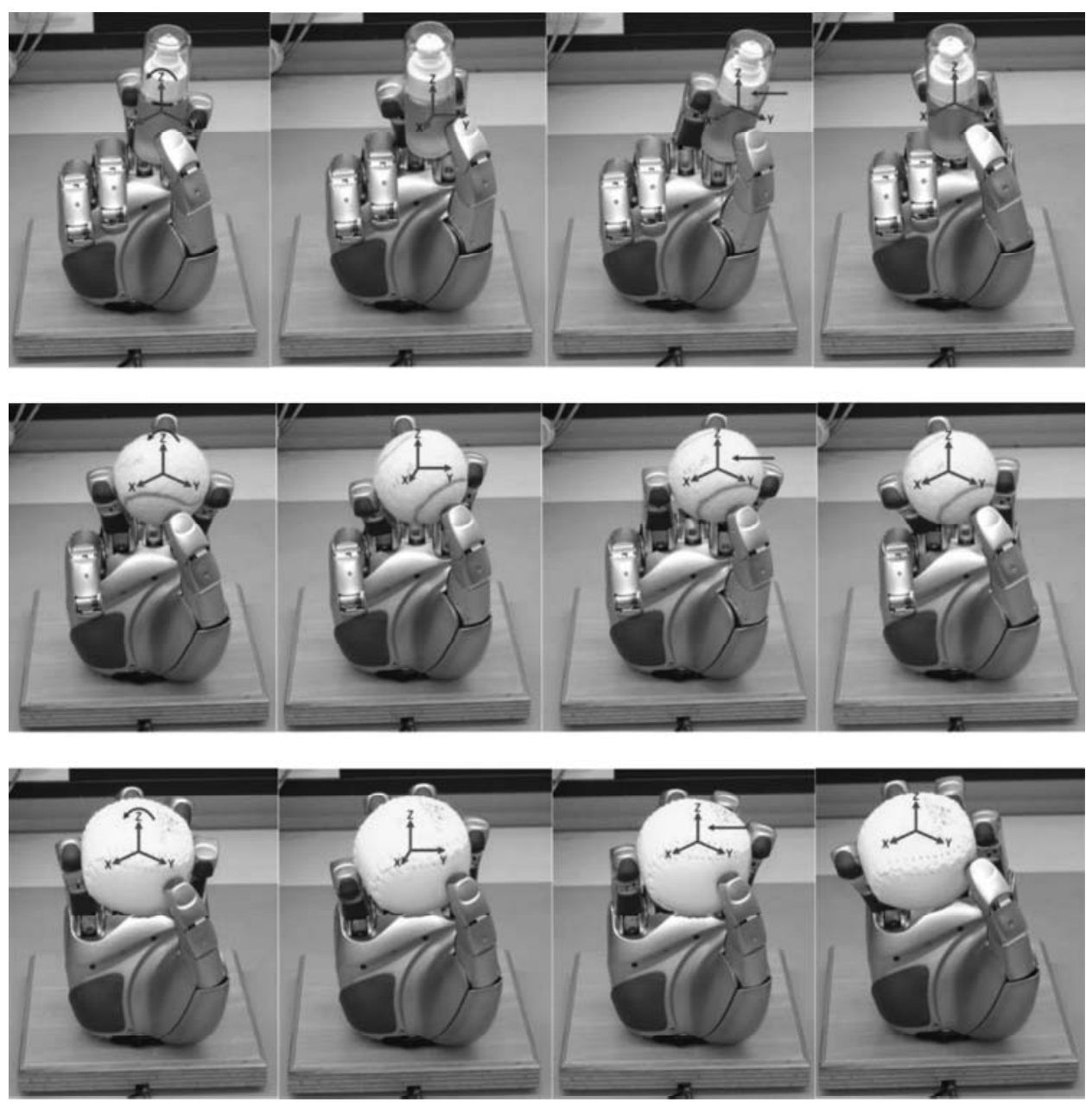

Figure 8. Rotation and translation experiments on 3, 4 and 5-fingered spatial impedance control.

with the environment. The position tracking errors, with and without friction and gravity compensation, are shown in Fig. 6, which prove the effectiveness of the proposed control and nonlinear compensation strategy.

In a further experiment, the robot pauses at virtual equilibrium position $x_{d}=[0.036,0.036,0.2]$ w.r.t Cartesian coordinates. The end point of the finger is pulled in one direction, then released. Figure 7 illustrates the corresponding Cartesian position offset and forces along the $x$-direction. The finger is able to overcome the gravity and friction, and returns towards to the desired Cartesian position as the robot finger is released. The static error 
Table 2

Control Parameters for Coordinated Spatial Impedance Control

\begin{tabular}{|l|c|c|c|c|c|c|c|}
\hline & $\begin{array}{c}K_{t} \\
\left(I_{3 \times 3} \cdot \mathrm{N} / \mathrm{m}\right)\end{array}$ & $\begin{array}{c}K_{o} \\
\left(I_{3 \times 3} \cdot \mathrm{Nm} / \mathrm{rad}\right)\end{array}$ & $\begin{array}{c}K_{c} \\
\left(I_{n \times n} \cdot \mathrm{N} / \mathrm{m}\right)\end{array}$ & $\Delta \gamma(\mathrm{rad})$ & $\Delta x_{o x}(\mathrm{~m})$ & $\begin{array}{c}\text { Object } \\
\text { Diameter }(\mathrm{mm})\end{array}$ & $\begin{array}{c}\text { Object } \\
\text { Weight }(\mathrm{g})\end{array}$ \\
\hline 3 Fingers & $2 \times 10^{3}$ & 2 & $4 \times 10^{2}$ & 0.7 & 0.02 & 35 & 100 \\
\hline 4 fingers & $2.3 \times 10^{3}$ & 2.5 & $4 \times 10^{2}$ & 0.6 & 0.02 & 65 & 86 \\
\hline 5 Fingers & $2.5 \times 10^{3}$ & 3 & $4 \times 10^{2}$ & 0.4 & 0.015 & 78 & 59 \\
\hline
\end{tabular}
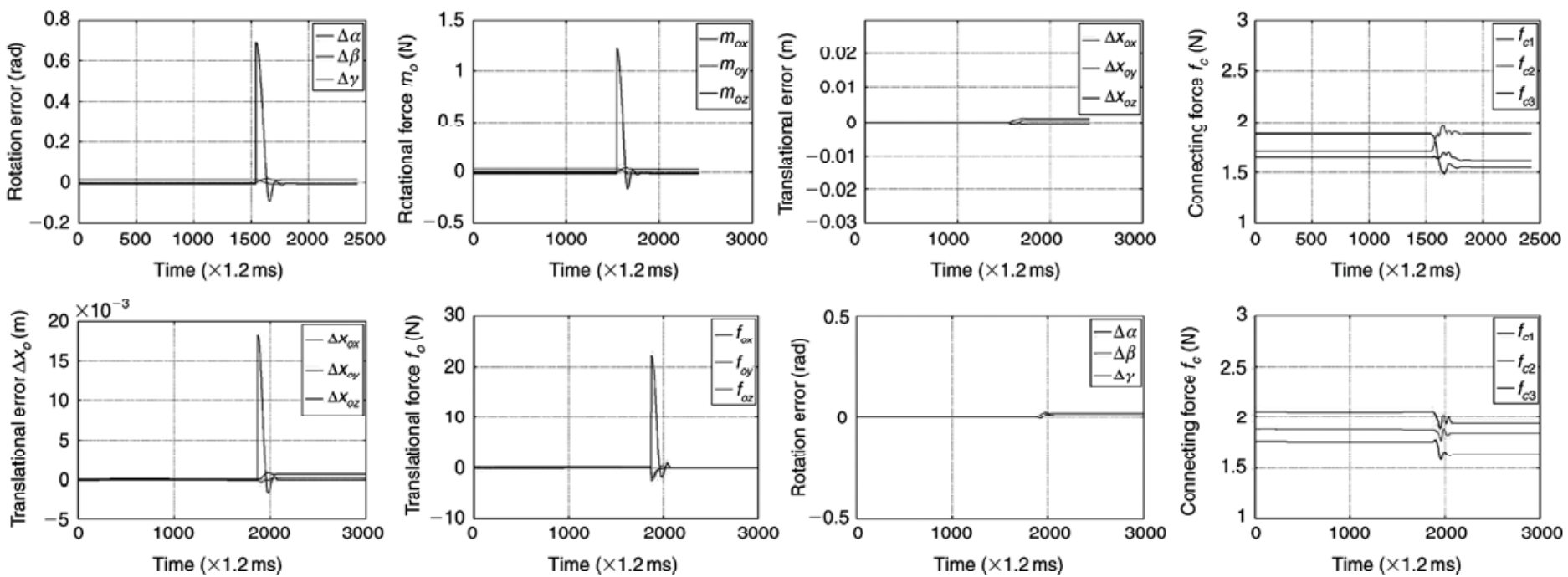

Figure 9. Experimental results of 3-fingered spatial impedance control.
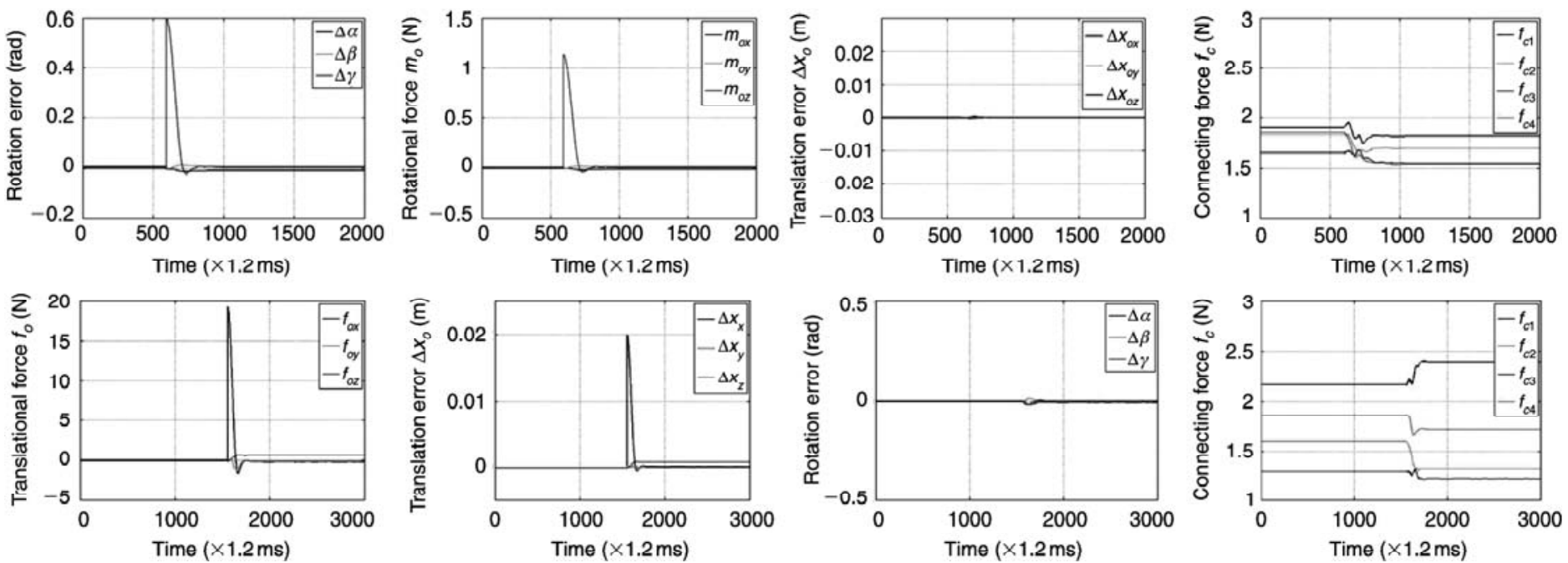

Figure 10. Experimental results of 4-fingered spatial impedance control.

along the $x$-axis is less than $0.2 \mathrm{~mm}$. Thus, the Cartesian impedance controller for the robot hand is successfully achieved with the proposed adaptive friction observer.

\subsection{Spatial Impedance Control}

Spatial impedance controller with nonlinear compensation is implemented on the robot hand in 3-, 4- and 5-finger configurations, as shown in Fig. 8. Control parameters are shown in Table 2, in which $I$ represents the identity matrix. $\Delta \gamma$ denotes a rotation step command (rotational difference with respect to the initial configuration) in Euler angle around the $z$-axis of the object frame. $\Delta x_{o x}$ is the translation step command (translational difference with respect to the initial configuration) along the $x$-axis of the object frame. To evaluate the coordinated spatial control law with the proposed object frame, object translation and rotation experiments are conducted on the robot hand. Specifications of the grasped objects are listed in Table 2.

The step response of the object rotation around the $z$-axis and translation along $x$-axis are shown in Figs. 9-11. There are high force peaks during the rotation and 

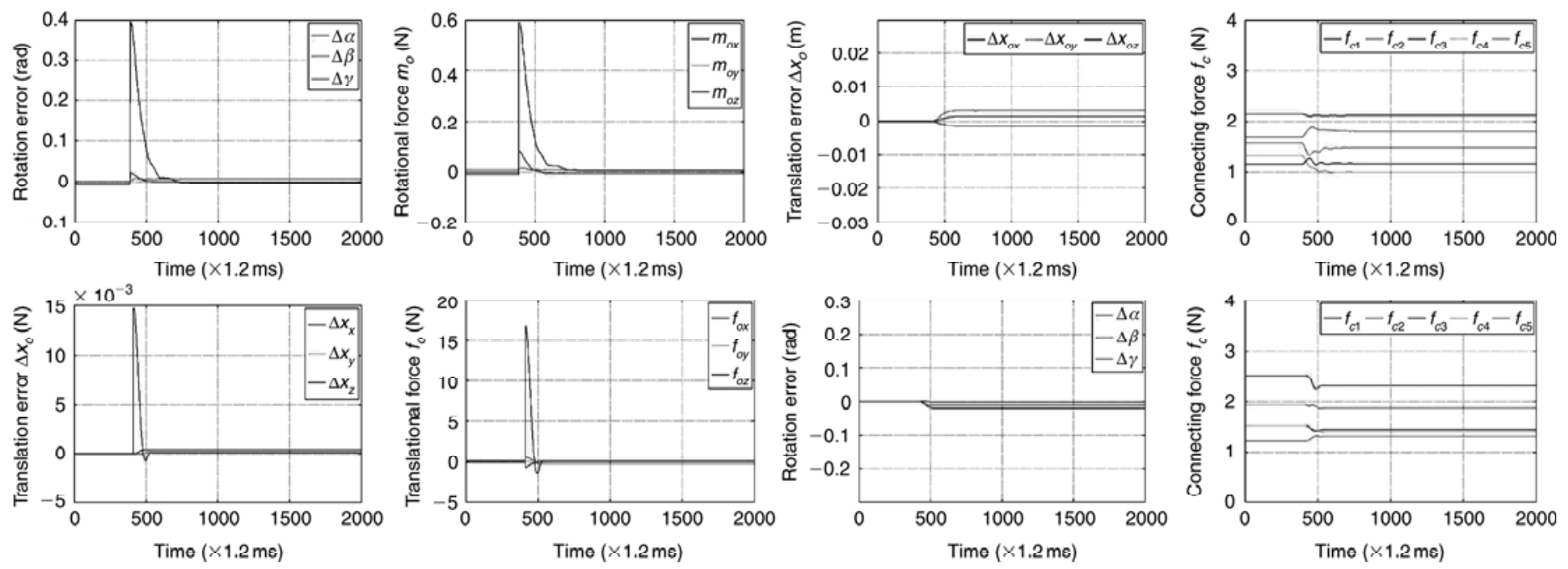

Figure 11. Experimental results of 5-fingered spatial impedance control.

translation, which can be observed in the experimental results. The steady state is achieved within $5 \mathrm{~ms}$ after the step command. Steady-state errors are less than $0.01 \mathrm{rad}$ for rotation, and less than $0.001 \mathrm{~m}$ for translation for 3-, 4- and 5-fingered spatial impedance control. Connecting forces of the thumb are larger than the other fingers in all the experiments, as the thumb opposes to the other fingers during grasping. This fact indicates that the configuration of the thumb should be considered more thoroughly both in the mechanical and controller design aspects, depending on different tasks.

By distributing the 6 -DoFs object driving forces generated by the controller to every single finger of the $n$-fingered robot hand with using the proposed object frame, $n$ fingers can work cooperatively to manipulate different objects. The overshooting decreases while increasing $n$ (fingers), as the driving load on each finger decreases. On the other hand, joint friction's influences increase on the performance of the controller as $\mathrm{n}$ increases, due to rising friction forces in comparison to the driving torque, which are treated as external forces by the controller. Although actual contact conditions do not completely correspond to the PCWF contact model, and both the object and fingertip are not ideally rigid, the modelling error can be compensated by the compliance behaviour of the spatial impedance controller [45].

\section{Conclusion}

In this work, experimental analysis for impedance control strategies in both Cartesian and object level are presented with the dexterous robot hand DLR-HIT II. An adaptive friction compensation approach based on the EKF is also proposed to address the highly nonlinear friction in the finger joints. The modular robot finger of the DLR-HIT II is modelled, for which an impedance controller with joint torque feedback in Cartesian space is implemented. Furthermore, a flexible $n$-fingered object frame is proposed in this work, applicable to any finger configuration of $n \geq 3$. Based on the proposed object frame, a coordinated spatial impedance controller is implemented on the DLR-HIT II hand. Stability of the closed-loop system with friction observer is analysed. Spatial and Cartesian impedance control experiments are conducted with the proposed friction observer, which improves position tracking performance as demonstrated in the experimental results.

\section{References}

[1] T. Mouri, H. Kawasaki, and K. Yoshikawa, Anthropomorphic robot hand: Gifu Hand III, Proc. Int. Conf. on Control, Automation and Systems, 2002, 1288-1293.

[2] J. Butterfass, M. Grebenstein, H. Liu, and G. Hirzinger, DLRHand II: next generation of a dextrous robot hand, Proc. IEEE Int. Conf. on Robotics and Automation, 2001, 109-114.

[3] H. Liu, P. Meusel, N. Seitz, G. Hirzinger, M. Jin, Y. Liu, and Z. Xie, The modular multisensory DLR-HIT-hand: hardware and software architecture, IEEE/ASME Transactions on Mechatronics, 13, 2008, 461-469.

[4] J.K. Salisbury and J.J. Craig, Articulated hands: force control and kinematic issues, International Journal of Robotics Research, 1, 1982, 4-17.

[5] S. Jacoben, J.E. Wood, D.F. Knutti, and K.B. Biggers, The Utah/MIT dexterous hand: work in progress, International Journal of Robotics Research, 3, 1984, 21-50.

[6] C. Lovchik and M. Dier, The robonaut hand: a dextrous robotic hand for space, Proc. IEEE Int. Conf. on Robotics and Automation, 1999, 907-912.

[7] M. Grebenstein, A. Albu-Schaffer, T. Bahls, M. Chalon, O. Eiberger, W. Friedl, R. Gruber, S. Haddadin, U. Hagn, R. Haslinger, H. Hoeppner, S. Joerg, M. Nickl, A. Nothhelfer, F. Petit, J. Reill, N. Seitz, T. Wimboeck, S. Wolf, T. Wuesthoff, and G. Hirzinger, The DLR hand arm system, Proc. 2011 IEEE Int. Conf. on Robotics and Automation (ICRA), 2011, 3175-3182.

[8] H. Liu, K. Wu, P. Meusel, N. Seitz, G. Hirzinger, M. Jin Y. Liu, S. Fan, T. Lan, and Z. Chen, Multisensory five-finger dexterous hand: the DLR/HIT Hand II, Proc. IEEE/RSJ Int. Conf. on Intelligent Robots and Systems, 2008, 3692-3697.

[9] N. Hogan, Impedance control - an approach to manipulation. I - Theory. II - Implementation. III - Applications, ASME Transactions, Journal of Dynamic Systems, Measurement, and Control, 107, 1985, 8-16.

[10] R.G. Bonitz and T.C. Hsia, Internal force-based impedance control for cooperating manipulators, IEEE Transactions on Robotics and Automation, 12, 1996, 78-89.

[11] S.A. Schneider and R.H. Cannon Jr, Object impedance control for cooperative manipulation: theory and experimental results, 
IEEE Transactions on Robotics and Automation, 8, 1992 383-394.

[12] C. Ott, A. Albu-Schaeffer, A. Kugi, S. Stramigioli, and G. Hirzinger, A passivity based Cartesian impedance controller for flexible joint robots - Part I: torque feedback and gravity compensation, Proc. IEEE Int. Conf. on Robotics and Automation, 2004, 2659-2665.

[13] A. Albu-Schaeffer, C. Ott, and G. Hirzinger, A passivity based Cartesian impedance controller for flexible joint robots - Part II: full state feedback, impedance design and experiments, Proc. IEEE Int. Conf. on Robotics and Automation, 2004, 2666-2672.

[14] L. Biagiotti, H. Liu, G. Hirzinger, and C. Melchiorri, Cartesian impedance control for dexterous manipulation, Proc. IEEE/RSJ Int. Conf. on Intelligent Robots and Systems, 2003, 3270-3275.

[15] R. Wei, X. Gao, M. Jin, Y. Liu, H. Liu, N. Seitz, R. Gruber, and G. Hirzinger, FPGA based hardware architecture for HIT/DLR hand, Proc. IEEE/RSJ Int. Conf. on Intelligent Robots and Systems (IROS 2005), 2005, 523-528.

[16] E.D. Fasse and J.F. Broenink, A spatial impedance controller for robotic manipulation, IEEE Transactions on Robotics and Automation, 13(4), 1997, 546-556.

[17] F. Caccavale, C. Natale, B. Siciliano, and L. Villani, Sixdof impedance control based on angle/axis representations, IEEE Transactions on Robotics and Automation, 15(2), 1999, 289-300.

[18] O. Khatib and J. Burdick, Motion and force control of robot manipulators, Proc. IEEE Int. Conf. on Robotics and Automation, 3, 1986, 1381-1386.

[19] S. Stramigioli, Modeling and IPC control of interactive mechanical systems: a coordinate-free approach (London: Springer Verlag, 2001).

[20] T. Wimböck, Ch. Ott, A. Albu-Schäffer, and G. Hirzinger, Comparison of object-level grasp controllers for dynamic dexterous manipulation, International Journal of Robotics Research, 31(1), 2011.

[21] C. Canudas de Wit, H. Olsson, K. Astrom, and P. Lischinsky, A new model for control of systems with friction, IEEE Transactions on Automatic Control, 40(3), 1995, 419-425.

[22] N. Kircanski and A. Goldenberg, An experimental study of nonlinear stiffness, hysteresis, and friction effects in robot joints with harmonic drives and torque sensors, The International Journal of Robotics Research, 16(2), 1997, 214.

[23] H. Olsson, Friction models and friction compensation, Control, 4(3), 1998, 1-37.

[24] H. Liu, Y. Liu, M. Jin, K. Sun, and J. Huang, An experimental study on cartesian impedance control for a joint torque-based manipulator, Advanced Robotics, 22(11), 2008, 1155-1180.

[25] C. Canudas de Wit and P. Lischinsky, Adaptive friction compensation with partially known dynamic friction model, International Journal of Adaptive Control and Signal Processing, 11, 1997, 65-80.

[26] H. Olsson and K.J. Astrom, Observer-based friction compensation, Proc. 35th IEEE of Decision and Control, 4, 1996, 4345-4350.

[27] B. Friedland and Y.J. Park, On adaptive friction compensation, IEEE Transactions on Automatic Control, 37(10), 1992, 16091612 .

[28] J. Huang, Z. Xie, H. Liu, and K. Sun, Adaptive Cartesian impedance control system for flexible joint robot by using DSP/FPGA architecture, International Journal of Robotics and Automation, 23(6), 2008, 243-251.

[29] T. Murakami, F. Yu, and K. Ohnishi, Torque sensorless control in multidegree-of-freedom manipulator, IEEE Transactions on Industrial Electronics, 40(2), 1993, 259-265.

[30] T. Takahashi, T. Tsuboi, T. Kishida, Y. Kawanami, S. Shimizu, M. Iribe, T. Fukushima, and M. Fujita, Adaptive grasping by multi-fingered hand with tactile sensor based on robust force and position control, Proc. IEEE Int. Conf. on Robotics and Automation (ICRA 2008), 2008, 264-271.

[31] Z. Chen, N. Lii, T. Wimböck, S. Fan, and H. Liu, Experimental evaluation of Cartesian and joint impedance control with adaptive friction compensation for the dexterous robot hand DLR-HIT II, International Journal of Humanoid Robotics, 8, 2011, 649-671.
[32] M. Spong, K. Khorasani, and P. Kokotovic, An integral manifold approach to the feedback control of flexible joint robots, IEEE Journal of Robotics and Automation, 3, 1987, 294-300.

[33] Z. Chen, N. Lii, T. Wimböck, S. Fan, M. Jin, C. Borst, and H. Liu, Experimental study on impedance control for the fivefinger dexterous robot hand DLR-HIT II, Proc. IEEE/RSJ Int. Conf. on Intelligent Robots and Systems, (IROS 2010), 2010, 5867-5874.

[34] Z. Chen, N. Lii, S. Fan, M. Jin, and H. Liu, Cartesian impedance control on five-finger dexterous robot hand DLRHIT II with flexible joint, Proc. IEEE Int. Conf. on Intelligent Robotics and Applications (ICIRA 2010), 2010, 1-12.

[35] B. Siciliano and O. Khatib, Springer handbook of robotics (Springer-Verlag Berlin Heidelberg: Springer-Verlag New York Inc., 2008), 166-167.

[36] A. Gelb, Applied optimal estimation (Cambridge, MA: MIT Press, 2002).

[37] R.G. Brown and P.Y.C. Hwang, Introduction to random signals and applied Kalman filtering (New York: Wiley, 1992).

[38] C.P. Connette, Intern report on DLR Hand II (Institute of Robotic hand Mechatronics, DLR, 2006).

[39] M.W. Spong, S. Hutchinson, and M. Vidyasagar, Robot modeling and control (Hoboken, NJ: John Wiley \& Sons, 2006).

[40] A. Kerner, The convergence of the extended Kalman filter, Directions in mathematical systems theory and optimization (Springer-Verlag Berlin Heidelberg: Springer, 2003), 173-182.

[41] K. Reif, S. Gunther, E. Yaz, and R. Unbehauen, Stochastic stability of the continuous-time extended Kalman filter, Proc. IEE Control Theory and Applications, 147, 2000, 45-52.

[42] Y. Song and J.W. Grizzle, The extended Kalman filter as a local asymptotic observer for discrete-time nonlinear systems, Journal of Mathematical Systems, Estimation and Control, 5, 1995, 59-78.

[43] M. Boutayeb, H. Rafaralahy, and M. Darouach, Convergence analysis of the extended Kalman filter used as an observer for nonlinear deterministic discrete-time systems, IEEE Transactions on Automatic Control, 42, 1997, 581-586.

[44] L. Le Tien, A. Albu-Schaeffer, A. De Luca, and G. Hirzinger, Friction observer and compensation for control of robots with joint torque measurement, Proc. IEEE/RSJ Int. Conf. on Intelligent Robots and Systems, IROS 2008, 2008, 3789-3795.

[45] M.R. Cutkosky, On grasp choice, grasp models, and the design of hands for manufacturing tasks, IEEE Transactions on Robotics and Automation, 5(3), 1989, 269-279.

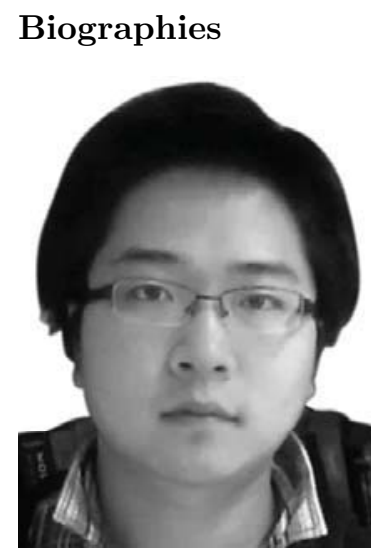

Zhaopeng Chen received the B.S. degree in mechanical engineering and automation, and the M.S. and Ph.D. degrees in mechatronic engineering, from the Harbin Institute of Technology, Harbin, China, in 2005, 2007 and 2012, respectively. From 2008 to 2010, he was a joint-train Ph.D. candidate in the Institute of Robotics and Mechatronics at the DLR German Aerospace Center, Oberpfaffenhofen, Germany. Since 2010, he has been with the DLR German Aerospace Center in Wessling, Germany, as a research scientist. His current interests include dexterous robot hands and non-linear control. 


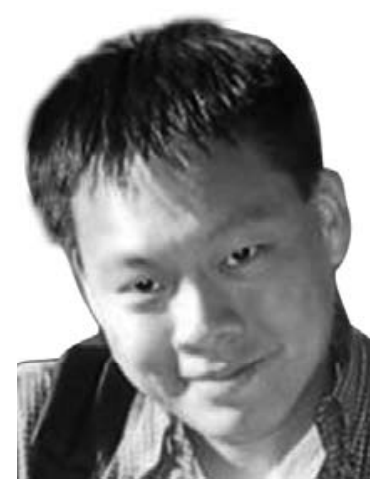

Neal Y. Lii received the B.S. degree in aeronautics and astronautics in 1995 from Purdue University, West Lafayette, IN, the M.S. degree in mechanical engineering in 1999 from Stanford University, Palo Alto, CA, and the Ph.D. degree in engineering (electrical engineering division) in 2009 from the University of Cambridge, Cambridge, UK. He has held industrial positions in North America and Europe working in the area of mechatronics. He worked on a variety of automotive applications ranging from driver assistance, powertrain control systems, and drive-bywire systems, the latter of which was carried out at BMW AG in Munich, Germany. He has been with the DLR German Aerospace Center in Wessling, Germany since 2009. His current projects include robotic endeffector design and applications, space robotics, and autonomous electrical vehicles.

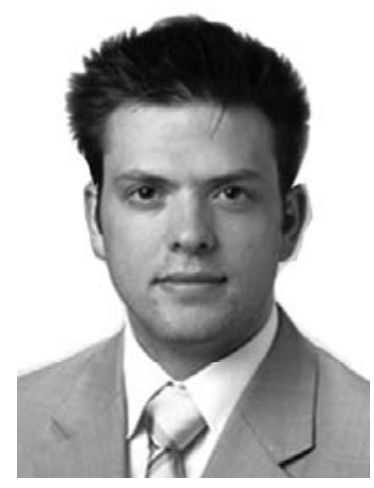

Thomas Wimböck studied electrical engineering at the Technical University of Munich (TUM) with visits to the Rensselaer Polytechnic Institute, Troy, New York, and to the École Polytechnique Fédérale de Lausanne, Switzerland. In 2004, he received his B.Sc. and M.Sc. degrees in electrical engineering from TUM. Subsequently, he joined the DLR, Institute of Robotics and Mechatronics, as a research scientist. His main research interests include nonlinear control, dexterous robot hands, impedance control, VS control, and two-handed (humanoid) manipulation. He received, together with his colleagues, several awards, including the Industrial Robot Outstanding Paper Award 2007, International Conference on Robotics and Automation Best Video Award 2007 and 2009.

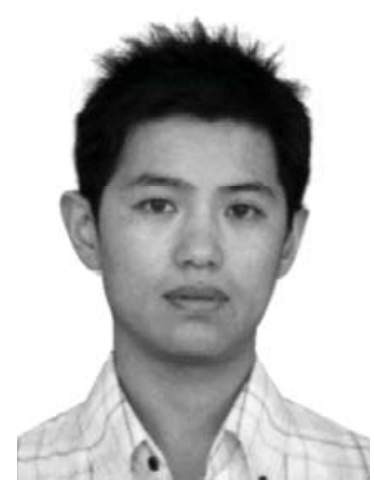

Shaowei Fan received the B.Eng, M.Eng., and Ph.D. degrees in mechatronics engineering from the Harbin Institute of Technology, Harbin, China, in 2001, 2005 and 2010, respectively. Since 2005, he has been with the State Key Laboratory of Robotics and System, Harbin Institute of Technology, where he currently holds the position of an associate researcher. His current research interests include anthropomorphic robot dexterous hands, prosthetics hands, tactile sensors, and the grasping synthesis.

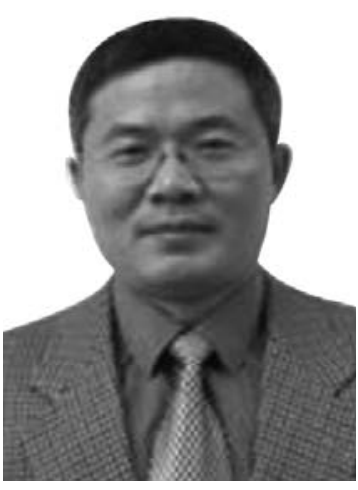

Hong Liu received the Ph.D. degree from the Harbin Institute of Technology, Harbin, China, in 1993. During 1991-1993, he was a joint-train Ph.D. candidate in the Institute of Robotics and System Dynamics, German Aerospace Research Establishment, Wessling, Germany, where, since 1993 , he has been a research fellow. His current research interests include the development of dexterous robot hands, space robotics, sensors, and control algorithms.

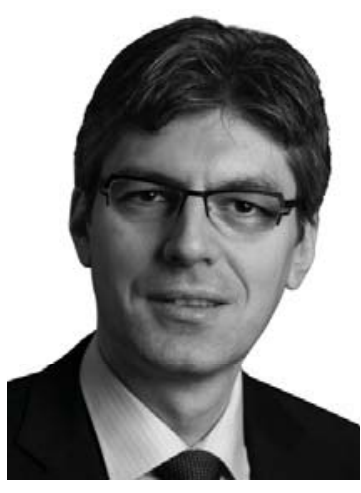

Alin Albu-Schäffer received his M.S. in electrical engineering from the Technical University of Timisoara, Romania, in 1993 and his Ph.D. in automatic controlfrom the Technical University of $\mathrm{Mu}$ nich in 2002. Since 2012, he has been the head of the Institute of Robotics and Mechatronics at the German Aerospace Center (DLR), which he joined in 1995 as a Ph.D. candidate. Moreover, he is a professor at the Technical University of Munich, holding the chair for "Sensor Based Robotic Systems and Intelligent Assistance Systems' in the Computer Science Department. His personal research interests include robot design, modelling and control, nonlinear control, flexible joint and variable compliance robots, impedance and force control, physical humanrobot interaction, and bio-inspired robot design and control. He received several awards, including the IEEE King-Sun Fu Best Paper Award of the Transactions on Robotics in 2012, several ICRA and IROS Best Paper Awards as well as the DLR Science Award. He was strongly involved in the development of the DLR light-weight robot and its commercialization through technology transfer to KUKA. 